\title{
Exploration and Research on Integrating Programming Education into Junior Middle School Mathematics Classroom
}

\author{
Yiqin $\mathrm{BAO}^{\mathrm{a}}$, Gui WANG ${ }^{\mathrm{a}}$ and Zhengtang SUN ${ }^{\mathrm{b}}$ \\ ${ }^{a}$ College of Information Engineering, Nanjing XiaoZhuang University, China \\ ${ }^{b}$ School of Computer Science and Technology, Heilongjiang University, China
}

\begin{abstract}
We are in the era of big data, and it is particularly important to cultivate students' data processing ability. Python is an important auxiliary tool for data analysis, and its syntax is concise and clear, which is suitable for junior high school students to start learning code language. Making full use of modern information technology and Innovating Curriculum form is the new requirement of "China education modernization 2035". Integrating Python into junior high school mathematics classroom is not only the innovation of mathematics curriculum form, but also a good way to cultivate students' thinking ability and programming thought. Based on the understanding of Python software and data processing plate in junior high school mathematics curriculum, this paper analyzes the promotion of students' thinking ability by integrating Python into junior high school mathematics classroom, and explores the strategies of applying Python in teaching combined with cases.
\end{abstract}

Keywords. Python, Junior high school mathematics, data processing

\section{Introduction}

With the advent of the era of big data and artificial intelligence, as an important way to cultivate students' digital skills and programming thinking, programming education has become the starting point of educational reform and deep cultivation [1]. In recent years, various researches on the development of students' programming thinking have been carried out continuously and integrated into the school classroom, which has become the mainstream trend of the development of students' Programming Education [2]. Li et al. [3] engaged in experimental teaching research on Mu class. Tsai et al. [4] improved students' interest in learning through flipping class. He et al. [5] conducted research on teaching mode exploration and practice. However, Junior middle school mathematics teaching focuses on cultivating students' thinking ability, and most of these studies are the research of teaching mode. Therefore, it is worth exploring and studying to integrate programming education into mathematics teaching and python into junior middle school mathematics curriculum.

This paper first introduces the characteristics of programming language, then studies the role of programming language Python in enhancing thinking ability, adding teaching interest and expanding students' vision, and finally summarizes the full text.

${ }^{1}$ Corresponding Author: Yiqin Bao; E-mail: baoyiqin@njxzc.edu.cn 


\section{Powerful programming language-Python}

Python is a popular programming language in recent years. It was developed by Guido van Rossum at the end of 1989. As a free and open source cross platform advanced dynamic programming language, Python supports command programming and functional programming, and fully supports object-oriented programming. Its syntax is simple and clear, and it provides a large number of built-in objects and functions, Becoming a pattern is very consistent with human thinking methods and habits [6].

The features of Python programming language are as follows:

1) Powerful function and simple operation: it has a very wide range of applications. At present, it has penetrated into almost all majors and fields such as computer science and technology, statistical analysis, computational visualization, mobile terminal development, graphics and image processing, artificial intelligence, etc. [7].

2) Data analysis database has complete functions: its own database has complete functions, such as NumPy and SciPy for the development of computer science industry, Django for the development of computer web pages, etc. [8]. These databases include many typical data models, which are easy to use directly when processing data and provide work efficiency [9].

3) Cross platform operation is simple and low cost: at this stage, Python can be well compatible with Linux, Windows and other platforms, and can run well [10].

4) Meet the preferences of the audience: in many programming languages, Python stands out with its own advantages and becomes one of the most commonly used programming software for software designer.

The future era is the era of AI, and Python is the closest language to AI. Therefore, Python language course will become a trend of children's learning. At present, Python language is gradually replacing VB language into middle school information technology curriculum. The primary problem of Python Programming Teaching in junior high school is how to stimulate students' interest in learning, and then integrate with mathematics learning to help students improve their thinking ability, and finally achieve the training goal of students' comprehensive quality [11-12].

\section{Introducing Python - Enhance thinking ability}

Programming is a kind of activity to investigate students' thinking comprehensively. Bill Gates once said: "learning programming can exercise your thinking, help you think better, and create a way of thinking that I think is very useful in all fields". In the process of teaching, the introduction of Python language and the creation of teaching situation will become an effective way to realize the modernization of mathematics education. This can not only help students consolidate their mathematical knowledge and optimize their knowledge structure, but also stimulate their thinking ability and help them master the correct thinking process and method, so as to cultivate their thinking ability of image, divergence and intuition.

\subsection{The influence of Python on the development of mathematical thinking}

Mathematical thinking depends on solid basic knowledge and skills, which is the thinking activity form of mathematical thinking and problem solving Junior high school students' thinking gradually from "experience type" to "theory type", which is the key period to cultivate students' mathematical thinking. At this moment, teachers 
should grasp the critical period and correctly guide students to promote their better development.

In junior high school mathematics curriculum, students have begun to contact with simple data processing knowledge - "data around us", "data concentration", "data dispersion", "simple application of Statistics" and other mathematical knowledge plates are reflected. During this period of study, students initially form the ability of data processing, but these abilities only stay at the theoretical level. Therefore, optimizing students' existing mathematical cognitive structure is the premise of cultivating mathematical thinking. In the "future education" system, curriculum and teaching are the most important carrier of education With the help of python, it will break the traditional course mode and enrich the classroom teaching content.

In the teaching of "3.4 variance" in the ninth grade mathematics volume I of Jiangsu Education Press, understanding the process of the generation and formation of the concept of variance is the key and difficult point of students' learning, and it is also the place where students are easily confused. Teachers in the classroom into Python can be a good solution to this problem. With the help of Python to draw the image of data, through the comparison of different groups of data graphs, we can feel the discrete degree of data in the gap between each group of data and its average value, so as to deeply understand the concept of variance and master mathematical knowledge. In this process, students actively participate in thinking and solving problems, which will gradually improve their mathematical thinking ability.

\subsection{The influence of Python on the development of logical thinking}

Logical thinking ability is a person's ability to think correctly and reasonably, that is, the ability to observe, compare, analyze, synthesize, abstract, generalize, judge and reason things, and the ability to use scientific logical methods to express his thinking process accurately and orderly. Relatively speaking, logical thinking ability can be more reflected in mathematics and other theoretical disciplines.

With the reform of the new curriculum, people attach great importance to the cultivation of students' logical thinking ability. Therefore, the cultivation of students' logical thinking ability has gradually become the top priority of teachers' Teaching. In the process of teaching, if teachers can take programming education to let students contact with programming education and gradually learn logical thinking, it will have a huge impact on students' lifelong development and social development, as well as on promoting the teaching progress of this subject.

Programming for logic rigor, thinking creativity is very high, its core is algorithm, and the ability of logical thinking in mathematics is very important. If Python is added to the teaching, students can gradually develop their logical thinking ability in code writing. For example, when "seeking mode" is implemented with Python, we need to understand the concept of mode and its formation process, that is, to accumulate the most frequent non repeated values in the data, then we can write correct and beautiful code.

\subsection{The influence of Python on the development of programming thinking}

With the rapid development of the times and driven by the trends of informatization, intelligence and integration, the cultivation of programming thinking becomes clear and important, and the core of programming thinking is computational thinking, that is, "understanding problems - the thinking process of finding paths". Programming ideas are influenced by computer language and computer operating environment to a certain 
extent.Therefore, programming thinking is not a commonly recognized programming skill, but an efficient way of thinking to solve any problem.

The new generation of artificial intelligence development plan issued in 2017 in China pointed out that artificial intelligence related courses should be set up in primary and secondary schools, and programming education should be gradually promoted. Python language as the most in line with the way of human thinking and habits of programming language into the school classroom is a very meaningful move, plays a directional role, bearing the goal of education.

At present, the computer has gradually become a pan social form of existence that affects the way of thinking and ability of human beings. In the junior high school mathematics classroom integrated with Python, students constantly use programming language in the process of teacher guidance, self practice and team exploration, so that children can imperceptibly cultivate programming thinking and solve problems more efficiently.

\section{Python into the classroom -- add fun and broaden horizons}

\subsection{Using Python to design statistical chart}

In junior high school mathematics data processing problems, Jiangsu Education Press seventh grade mathematics volume II first began to contact the knowledge of frequency, the most common topic is to calculate the frequency distribution table and draw the frequency distribution map according to the data. If we learn Python, we can better grasp the situation of the data, at the same time, we can check the answers of the written calculation.

Example 1: input a string, convert it into uppercase, count the times of each letter, and draw the statistical histogram.

1) The Python program is as follows:

$\mathrm{a}=$ input("Please enter character string:")

$\mathrm{b}=$ list(a.upper())

$\mathrm{c}=\{\}$

for $\mathrm{i}$ in $\mathrm{b}$ :

$\mathrm{c}[\mathrm{i}]=\mathrm{b} \cdot \operatorname{count}(\mathrm{i})$

$\mathrm{x}=\operatorname{list}(\mathrm{c} . \operatorname{keys}())$

$\mathrm{y}=\operatorname{list}($ c.values ()$)$

\#Draw histogram

plt.bar(np.arange $(0$, len $(x), 1)$, height $=y)$

plt.xticks(np.arange $(0$, len $(x), 1), x)$

plt.xlabel("Upper case letters")

plt.ylabel("Numbers")

plt.title('Statistical histogram')

plt.show()

2) The results are as follows

If input: ABACsDEFcJGcFScwSJfLwPEWRFZZ

Then count the number of times each letter appears: $\left\{\right.$ A $^{\prime}: 2$, , $^{\prime}$ ': 1, 'C': 4, 'S': 3, 'D': 1, 'E': 2, 'F': 4, 'J': 2, 'G': 1, 'W': 3, 'L': 1, 'P': 1, 'R': 1, 'Z': 2 \}

The statistical histogram is as follows: 


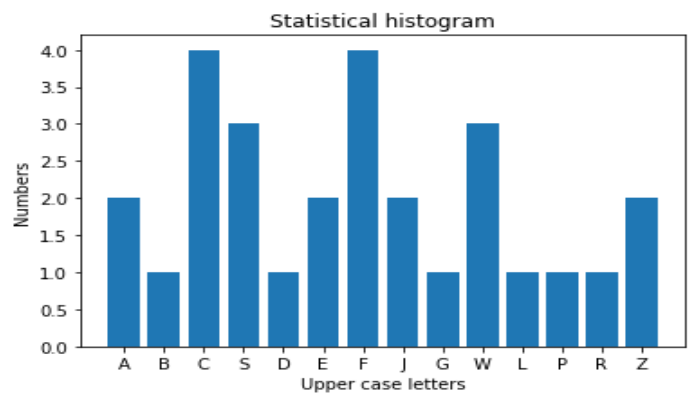

Figure 1. Histogram of letter occurrence times

\subsection{Using Python to solve statistics}

Mathematics teaching should be based on a solid foundation and impart knowledge through effective mathematics teaching methods. When junior high school students have a certain basic knowledge of probability, they will start to contact the knowledge of data, introduce basic statistics such as mean, median and variance, and require students to be able to solve them simply.If we use Python to help them learn with a good foundation of written calculation, it will stimulate students' desire to explore and improve their learning efficiency.

Example 2: Xiao Ming opened a grocery store. The following is his profit record in the past ten days (yuan): [85,98,76,98126138100,98106117]. Request the average, standard deviation and median of this group.

1) The Python program is as follows:

\#Raw Data

data $=[85,98,76,98,126,138,100,98,106,117]$

\#Calculate average value

average $=$ float $(\operatorname{sum}($ data $)) /$ len $($ data $)$

\#Calculate Standard deviation

total $=0$

for value in data:

total $+=($ value - average $) * * 2$

stddev $=$ math.sqrt $($ total $/$ len $($ data $))$

\#Calculate Median

data.sort()

$\operatorname{mid}=\operatorname{int}($ len(data $) / 2)$

if len(data) $\% 2==0$ :

median $=(\operatorname{data}[\operatorname{mid}-1]+$ data $[$ mid $]) / 2.0$

else:

median $=\operatorname{data}[\mathrm{mid}]$

print(average, stddev, median)

2) The results are as follows

Average: 98, Standard deviation: 17.55448660599335, Median: 99.0

In the above solving process, we use Python to demonstrate examples and solve problems, stimulate students' interest in learning, strengthen students' understanding of the principle of statistics solving, so as to master how to use these statistics to solve problems in combination with practice. 


\section{Conclusions}

In the teaching process of junior high school mathematics data processing plate, students may have a certain interest in contacting new knowledge at the initial stage, teachers can explain some examples and lead students to practice, which will stimulate students' interest in learning. This can strengthen students' understanding of the principle of statistical solution in the code writing, so as to achieve the effect of learning knowledge transfer, and then cultivate students' thinking ability.

Students can creatively use data information and data knowledge to solve problems, and find the beauty of mathematics in life in this process, so as to further improve students' interest in mathematics, and then potentially promote moral and moral education Attitude, emotion and other goals.

\section{Acknowledgement}

This work is supported by Key topics of the '13th five-year plan' for Education Science in Jiangsu Province (B-b /2020/01/18).

\section{References}

[1] Zhang Jinbao, Ji Lingyan. The evolution of the orientation of information technology education in primary and secondary schools [J]. Audio visual education research, 2018 (5): 108-114.

[2] Sun Lihui, Liu Siyuan, Li Manman. Action path of children's programming education in the era of artificial intelligence -- Based on the survey report of "necessary conditions for the development of children's programming education" in Japan [J]. Audio visual education research, 2019,40 (08): $114-120+128$.

[3] Xianyi Li, Senlin Tong, Xiaoying Liu. Experimental teaching preview system based on Mu class concept. Laboratory science, 2017, 20 (5): 161-163.

[4] Tina Pingting Tsai, Jyhjong Lin, Chingsheng Hsu, "Using ePUB3 eBook-based SNS to Enhance Students , Learning Effects in Flipped Classes," Journal of Internet Technology, 2020(12),21(7):1999-2008.

[5] HE Shi-tian,TAN De-li.Exploration and practice of "principles of computer composition" teaching mode based on synergy inside and outside class[J]. Computer Engineering \& Science. 2019(12),41(1):144-148.

[6] Tao Wenling, Hou Dongqing. Discussion on Python application based on problem solving -- Taking Python batch processing excel file as an example [J]. Journal of Ezhou University, 2019,26 (01): $107-109+112$.

[7] Xiao Le, Cong Tianwei, Yan Wei. Web big data collection and data analysis based on Python [J]. Computer knowledge and technology, 2018,14 (22): 9-11.

[8] Wang Liang, Zuo Wentao. Research on the application of Python programming language in big data collection and analysis [J]. Computer products and circulation, 2020 (01): 22.

[9] Xu Dongsheng, Chen Hongxia. Research on using Python to promote agricultural product information efficiency and application willingness under the background of big data [J]. Rural economy, 2018 (05): 99-103.

[10] Xia huosong, pan Xiaoting. Research on the relationship between big data academic research and talent demand based on Python mining [J]. Journal of information resource management, 2017,7 (01): 4-12.

[11] Chen Xiaojuan. Learning by doing: a case study of Python program [J]. China educational technology equipment, 2019 (03): 98-99+ 102.

[12] Qiu Yu, Shang Zhi Nian. Practice and thinking of Python program teaching in junior high school based on micro: Bi [J]. China information technology education, 2018 (Z3): 52-55. 